\title{
Diagnóstico de vaginosis bacteriana en un consultorio de planificación familiar de la Región Metropolitana, Chile
}

\author{
Eduardo Lillo G., Sandra Lizama I., Jorge Medel C. y M. Angélica Martínez T.
}

\section{Diagnosis of bacterial vaginosis in women attending a family planning clinic in the Metropolitan Region of Chile}

In order to determine the prevalence of bacterial vaginosis (BV) among women attending a family health Center and to evaluate the accuracy of Amsel's diagnostic procedure relative to Nugent scoring for diagnosing this condition, we obtained vaginal specimens from 100 women under 50 years attending a FPC from April to November of 2006. Women were enrolled consecutively on a schedule basis and were not selected by symptoms of genital infection. VB was diagnosed in $32 \%$ women, without significant differences by age $(p=0,114) .87 .5 \%$ women declared one sexual partner in the last 6 months. The Amsel's method had a sensitivity of $62.1 \%$, and a specificity of $92.3 \%$, with positive and negative predictive values of $81.8 \%$ and $83.3 \%$ respectively, being the presence of clue cells the most accurate parameter. Nevertheless, the lack of microscopes in the clinical practice limits the use of the Amsel's criteria. In conclusion, VB is a prevalent infection in the study population, being not associated with age or sexual activity. Diagnosis should be performed by the Nugent method.

Key words: Bacterial vaginosis, family planning.

Palabras clave: Vaginosis bacteriana, consultorio, planificación familiar.

\section{Introducción}

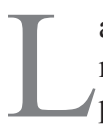

a vaginosis bacteriana (VB) es la infección vaginal más frecuente en el mundo, constituyendo un problema de salud pública por su asociación con patología obstétrica y ginecológica y el riesgo significativo de adquirir infecciones de transmisión sexual (ITS) ${ }^{1-5}$. En Chile, su prevalencia varía entre 27 y $32 \%$, cifra que se duplica en mujeres con parto prematuro o enfermedad inflamatoria pélvica ${ }^{6-10}$.

Dada su alta prevalencia, se recomienda tratar solamente las pacientes sintomáticas, las que representan $\sim 50 \%$ de los casos, como asimismo a mujeres embarazadas con riesgo de parto prematuro, siendo por ello necesario contar con una metodología de diagnóstico de $\mathrm{VB}^{1}$.

Se han propuesto distintos procedimientos para el diagnóstico de VB, siendo el método de Amsel y cols, que utiliza mayoritariamente parámetros clínicos, y el método de Nugent y cols, que se basa en parámetros microbiológicos, los más utilizados en la actualidad ${ }^{12-13}$. El método de Amsel, empleado frecuentemente en la práctica clínica, consiste en investigar cuatro parámetros en la secreción vaginal, estableciéndose el diagnóstico ante la presencia de al menos tres ${ }^{12}$. Estos parámetros son: secreción vaginal homogénea, adherente y grisácea, aumento del $\mathrm{pH}$ vaginal $(>4,5)$, producción de aminas volátiles de mal olor y presencia de "clue cells" en el examen microscópico. El método de Nugent se basa en cuantificar tres morfotipos bacterianos en el examen directo de la secreción vaginal teñido con Gram: bacilos grampositivo largos (Lactobacillus spp); cocobacilos Gram variable o gramnegativos que corresponden a Gardnerella vaginalis y Prevotella spp, respectivamente; y bacilos curvos Gram variable, que representan a Mobiluncus $\mathrm{spp}^{13}$.

No obstante que la VB es una infección frecuente en nuestro país, sólo existe una publicación que compara la eficiencia diagnóstica de los métodos de Amsel y Nugent en la práctica clínica?

Los objetivos de este estudio fueron determinar la prevalencia de VB y evaluar la eficiencia diagnóstica de los parámetros clínicos de Amsel, utilizando el método de Nugent como procedimiento de referencia para el diagnóstico de VB.

\section{Pacientes y Métodos}

Pacientes y muestras clínicas. Se invitó a participar a mujeres que consultaron por planificación familiar, entre abril y noviembre de 2006 en el Consultorio Santa Anselma, SSMO. Los criterios de inclusión fueron edad 15 a 49 años, abstinencia sexual de al menos 48 hrs y aprobación
Facultad de Medicina, Universidad de Chile, Santiago.

Escuela de Obstetricia y Puericultura (ELG, SLI, JMC).

Instituto de Ciencias Biomédicas Programa de Microbiología y Micología (MAMT).

Recibido: 19 de junio de 2009 Aceptado: 15 de abril de 2010

Correspondencia a: M. Angélica Martínez Tagle mamartin@med.uchile.cl 
del consentimiento informado. Los criterios de exclusión fueron embarazo, menstruación al momento de la toma de muestra, tratamiento antimicrobiano en los últimos 30 días, inmunosupresión o tratamiento con fármacos inmunosupresores. No se hizo selección por presencia o ausencia de signos o síntomas de infección vaginal y las pacientes fueron enroladas en forma consecutiva en un horario predeterminado. En cada oportunidad se aplicó un instrumento de recolección de datos clínicos, se efectuó examen físico de los genitales externos y se tomó muestras de secreción vaginal para los procedimientos de diagnóstico: se tomaron dos muestras con tórula estéril, las que fueron utilizadas para la detección de aminas volátiles y examen microscópico directo. Para el examen microscópico, la tórula fue extendida en un portaobjetos destinado a tinción de Gram y luego inoculada en $1 \mathrm{ml}$ de solución salina estéril para efectuar examen microscópico al fresco. Las muestras fueron obtenidas con uso de espéculo del fondo de saco vaginal.

\section{Diagnóstico de vaginosis bacteriana}

Método de Amsel. Se efectuó diagnóstico de VB en presencia de al menos tres de los siguientes cuatro criterios: $\mathrm{pH}$ vaginal $>4,5$, prueba de aminas positiva, observación de "clue cells" y presencia de secreción

Tabla 1. Prevalencia de vaginosis bacteriana según edad en 100 mujeres en planificación familiar. Consultorio Santa Anselma, Santiago de Chile, abril - noviembre de 2006

\begin{tabular}{|ccc|}
\hline $\begin{array}{c}\text { Edad } \\
\text { (años) }\end{array}$ & $\begin{array}{c}\text { Total pacientes } \\
\text { en el rango de edad }\end{array}$ & $\begin{array}{c}\mathbf{n}(\%) \\
\text { muestras positivas }\end{array}$ \\
\hline $15-20$ & 10 & $5(50)$ \\
$21-30$ & 23 & $6(26,1)$ \\
\hline $31-40$ & 40 & $12(30)$ \\
\hline $41-49$ & 27 & $9(33,3)$ \\
\hline Total & 100 & $32(32)$ \\
\hline
\end{tabular}

Tabla 2. Distribución de casos sintomáticos de vaginosis bacteriana según edad. Consultorio Santa Anselma,

Santiago de Chile, abril-noviembre de 2006

\begin{tabular}{|ccc|}
\hline Rango etario (años) & n mujeres con VB & n (\%) con signos o síntomas \\
\hline $15-20$ & 5 & $1(20)$ \\
$21-30$ & 6 & $5(83,3)$ \\
$31-40$ & 12 & $6(50)$ \\
$41-49$ & 9 & $7(77,8)$ \\
\hline Total & 32 & $19(59,4 \%)$ \\
\hline
\end{tabular}

vaginal adherente, homogénea, grisácea. $\mathrm{El} \mathrm{pH}$ de la secreción vaginal fue determinado del introito vaginal con una cinta de papel $\mathrm{pH}$ (Merck) con rango de $\mathrm{pH}$ de 3-6. Se consideró $\mathrm{pH}$ vaginal normal a un valor $\leq 4,5$ y alcalino a un $\mathrm{pH}>4,5$. La detección de aminas fue efectuada agregando una gota de $\mathrm{KOH}$ al $10 \%$ a una tórula con secreción vaginal. Una prueba positiva estuvo determinada por el desprendimiento inmediato de olor a pescado tras la aplicación de $\mathrm{KOH}$ y una prueba negativa al no desprendimiento de olor. La presencia de "clue cells" fue determinada en el examen microscópico al fresco, considerándose positivas aquellas muestras que presentaron células vaginales cubiertas de bacterias, en forma aislada o en grupos.

Método de Nugent. Para el diagnóstico de VB se evaluó el examen microscópico directo de la muestra vaginal mediante tinción de Gram, utilizando los criterios de Nugent y cols ${ }^{13}$.

Análisis estadístico. Los cálculos de sensibilidad, especificidad y valores predictores positivo (VPP) y negativo (VPN) fueron calculados según sus fórmulas habituales ${ }^{14}$. Las diferencias entre proporciones fueron evaluadas mediante la prueba de chi cuadrado $\left(\chi^{2}\right)$. Se consideró estadísticamente significativo a un error tipo $\alpha \leq 0,05$.

\section{Resultados}

De un universo de 1.262 mujeres en control y planificación familiar en el consultorio Santa Anselma, se enrolaron en el estudio a 100 de ellas. Empleando el método de Nugent como técnica de referencia, en 32 casos (32\%) se efectuó diagnóstico de VB, en 57 mujeres $(57 \%)$ la microbiota fue clasificada como normal y en 11 casos (11\%) como microbiota intermedia. Noventa y seis mujeres declararon la actividad sexual en los últimos 6 meses. De ellas, 84 mujeres $(87,5 \%)$ declararon pareja sexual única, $3(3,1 \%)$ señalaron dos parejas sexuales y $9(9,4 \%)$ indicaron abstinencia sexual.

En la Tabla 1 se presenta la frecuencia de VB según edad. No se observó diferencias significativas en la frecuencia de VB según grupo etario $(\mathrm{p}=0,114)$.

En la Tabla 2 se muestra la frecuencia de signos y síntomas de VB según edad. Diecinueve $(59,4 \%)$ de 32 mujeres con VB presentaron signos o síntomas de VB, pero no se observaron diferencias significativas por edad $(\mathrm{p}=0,054)$.

En la Tabla 3 se muestran los resultados de los procedimientos de Amsel y Nugent para el diagnóstico de VB. En 94 pacientes se dispuso de la totalidad de los parámetros clínicos para la comparación de ambos 


\begin{tabular}{|c|c|c|c|}
\hline \multirow[t]{2}{*}{ Amsel y cols } & \multicolumn{2}{|c|}{$\begin{array}{l}\text { Nugent y cols } \\
\text { n de muestras }\end{array}$} & \multirow[b]{2}{*}{ Total } \\
\hline & Positivo & Negativo & \\
\hline Positivo & 18 & 5 & 23 \\
\hline Negativo & 11 & 60 & 71 \\
\hline Total & 29 & 65 & 94 \\
\hline
\end{tabular}

métodos. La concordancia entre ambos procedimientos fue 78/94 (83\%), detectándose 11/29 (37,9\%) resultados falsos negativos y $5 / 65(7,7 \%)$ resultados falsos positivos por el método de Amsel.

En la Tabla 4 se muestran los valores de sensibilidad, especificidad, valores predictor positivo (VPP) y negativo (VPN) del método de Amsel y de sus parámetros individuales. El método de Amsel demostró una buena especificidad, 92,3\%, pero su sensibilidad alcanzó solamente $62,1 \%$, mientras que sus VPP y VPN fueron respectivamente 81,8 y $83,3 \%$. La determinación de "clue cells" fue el mejor parámetro individual, con $100 \%$ de especificidad y $97 \%$ de sensibilidad. La presencia de flujo vaginal sugerente de VB fue el parámetro menos sensible, $41,4 \%$, pero tuvo buena especificidad, $91 \%$. La demostración de aminas volátiles tuvo baja sensibilidad, $58,1 \%$, con buena especificidad, $88 \%$. La presencia de $\mathrm{pH}$ alcalino, ocupó el segundo lugar en sensibilidad, 72,4\% de sensibilidad, pero sólo alcanzó $65 \%$ de especificidad.

Con respecto a las categorías de Nugent, se observó el morfotipo correspondiente a Mobiluncus spp, en 9/32 $(28,1 \%)$ muestras clasificadas como VB y en ninguna mujer con microbiota normal o intermedia $(p<0,001)$.

\section{Discusión}

Por tratarse de una infección polimicrobiana, los métodos de elección para el diagnóstico de VB son los criterios clínicos de Amsel, que reflejan la fisiopatología de la VB o la demostración del desequilibrio en la microbiota vaginal mediante tinción de Gram. Cualquier procedimiento basado en la demostración de un agente asociado a VB en particular, ya sea por cultivo o técnicas moleculares no cuantitativas, tiene una baja especificidad, ya que estas bacterias forman parte de la microbiota normal.

La frecuencia de VB encontrada en este estudio es alta, resultando similar al observado en una población de mujeres que recibieron atención en un programa de planificación familiar en la Octava Región, 31,8\% ${ }^{8}$. Nuestros hallazgos sugieren que en la población estudiada, la VB no se asocia con conductas de riesgo de ITS ya que

\begin{tabular}{|l|lcccc|}
$\begin{array}{r}\text { Tabla 4. Sensibilidad, especificidad, valor predictor positivo (VPP) y } \\
\text { negativo (VPN) de los parámetros clínicos de Amsel para el } \\
\text { diagnóstico de vaginosis bacteriana }\end{array}$ \\
\begin{tabular}{|l} 
n muestras \\
analizadas
\end{tabular} & $\begin{array}{c}\text { Sensibilidad } \\
\text { (\%) }\end{array}$ & $\begin{array}{c}\text { Especificidad } \\
\text { (\%) }\end{array}$ & $\begin{array}{c}\text { VPP } \\
\text { (\%) }\end{array}$ & $\begin{array}{c}\text { VPN } \\
\text { (\%) }\end{array}$ \\
\hline Amsel* & 94 & 62,1 & 92,3 & 81,8 & 83,3 \\
\hline Flujo alterado & 94 & 41,4 & 90,8 & 66,7 & 77,6 \\
\hline pH vaginal > 4,5 & 94 & 72,4 & 64,6 & 47,7 & 84,0 \\
\hline Presencia de aminas & 96 & 58,1 & 87,7 & 69,2 & 81,4 \\
\hline $\begin{array}{l}\text { Presencia de } \\
\text { "clue cells" }\end{array}$ & 100 & 96,9 & 100 & 100 & 98,6 \\
\hline * $\geq 3$ parámetros positivos. VPP: Valor predictor positivo. VPN: Valor predictor negativo. & \\
\hline
\end{tabular}

sólo 3,1\% de las mujeres en que se tabuló la información habían tenido dos parejas sexuales en los últimos meses. Sería importante investigar en esta población factores bio-psicosociales como posibles factores de riesgo de VB. Más aún, un tercio de mujeres con VB tenía el morfotipo Mobiluncus, señalando los puntajes más altos de Nugent y con ello de una profunda alteración del ecosistema vaginal. Esta grave alteración podría indicar una demora en consultar por molestias vaginales, o sencillamente diferencias poblacionales. Un estudio global de salud reproductiva y perinatal demostró diferencias significativas en la prevalencia de Mobiluncus al Gram en distintas regiones geográficas ${ }^{15}$. La alta prevalencia detectada en nuestro estudio resulta muy superior a la detectada en países de Europa y es similar a la detectada en Zimbabwe ${ }^{15}$.

Con respecto a VB en mujeres embarazadas, Ovalle y cols, estudiaron muestras vaginales de 156 pacientes, 82 con riesgo de presentar infección genito-urinaria y 74 sin riesgo ${ }^{6}$. El estudio fue realizado en la policlínica de Alto Riesgo del Hospital San Borja Arriarán y en dos consultorios dependientes del SSMC, respectivamente, detectándose VB en 18/82 (22,0\%) mujeres embarazadas con riesgo y en $19 / 74(25,7 \%)$ sin riesgo, diferencia que no fue significativa ${ }^{6}$. Otro estudio nacional demostró 20,8\% de VB en la policlínica de Alto Riesgo del Hospital San Juan de Dios, confirmando una menor frecuencia de VB en mujeres embarazadas, probablemente por un factor hormonal de protección ${ }^{7}$.

Un buen diagnóstico de VB es necesario para definir apropiadamente el uso de antimicrobianos. El diagnóstico de VB por el método de Amsel ha demostrado ser útil, pero tiene dificultades en la medición y/o estandarización de los parámetros individuales, lo que le otorga variación interpersonal $^{16-18}$.

Los resultados de este y otros estudios señalan la determinación de "clue cells", el parámetro de mayor sensibilidad y especificidad entre los criterios de Amsel ${ }^{19-20}$. 
El método de Amsel no considera la cuantificación de las "clue cells", mientras que en una propuesta, Eschenbach y cols, consideran positivo a un porcentaje $\geq 20 \%$ de "clue cells" respecto de células epiteliales. Ambos puntos de corte se emplean indistintamente ${ }^{16}$.

La VB no es una infección de la secreción vaginal, sino que involucra la formación de biopelículas sobre la mucosa vaginal ${ }^{21}$. Las "clue cells" corresponden a fragmentos de distinto tamaño desprendidos de la biopelículas. Al interior de estos islotes celulares, la mayoría de las células corresponden a la descripción de una "clue cell".

Con relación al $\mathrm{pH}$ vaginal normal, este varía entre 3,8 y 4,1 en mujeres en edad fértil, mientras que en pacientes con $\mathrm{VB}$, producto de su alcalinización por las aminas producidas por las bacterias anaerobias, el $\mathrm{pH}$ es $>4,5^{12}$. Diferentes grupos han considerado distintos puntos de corte para $\mathrm{pH}$ en sus publicaciones; $\geq 4,5^{7,20}$, $>4,5^{19-20,22}, \geq 4,7^{15}$, otorgando a este parámetro una amplia variabilidad en sensibilidad (66,7 a $96 \%$ ) y especificidad $(28,8 \text { a } 91,1 \%)^{8,16,19-23}$. En este estudio, el $\mathrm{pH}$ tuvo $72,4 \%$ de sensibilidad y $64,6 \%$ de especificidad, siendo su mejor parámetro estadístico el VPN, con $84 \%$.

La detección de mal olor en la secreción vaginal es producto de la volatilización de las aminas producidas por los anaerobios y, aunque constituye el parámetro de menor sensibilidad de Amsel, es uno de los más específicos ${ }^{8,19-20,22}$.

A más de 50 años de su descripción por Gardner y Dukes, la VB continúa siendo un desafío clínico y microbiológico ${ }^{24}$. El término VB se define actualmente como el reemplazo del predominio de Lactobacillus spp que caracteriza a la microbiota vaginal normal, por un aumento en el crecimiento y concentración de Gardnerella vaginalis, algunas bacterias anaerobias estrictas: Prevotella spp, Bacteroides spp, Peptostreptococcus spp, Atopobium vaginae y Mobiluncus spp, conjuntamente con Mycoplasma hominis ${ }^{10,17-18}$. Con la pérdida del predominio de Lactobacillus spp se pierde su efecto protector en el nicho vaginal. No obstante, se han encontrado diferencias raciales en la estructura y composición de la microbiota vaginal, con la demostración de 12 clases de comunidades bacterianas en una población de 144 mujeres sanas asintomáticas en E.U.A. ${ }^{25}$. El rol de producir y mantener el $\mathrm{pH}$ ácido vaginal, tradicionalmente atribuido a Lactobacillus spp., puede ser efectuado por otras bacterias ácido lácticas, las que constituyen un grupo heterogéneo de géneros con un mayor predominio en mujeres de color. Estas comunidades bacterianas tendrían distinta capacidad de resiliencia a agresiones en el ecosistema vaginal, lo que podría relacionarse con la mayor prevalencia de VB que se observa en poblaciones de color en E.U.A. ${ }^{25}$. En lo que respecta al diagnóstico de $\mathrm{VB}$, esto podría explicar, porqué mujeres asintomáticas son catalogadas como portadoras de VB al Gram, al no presentar el morfotipo lactobacilar.

En conclusión, de acuerdo a este estudio la VB pareciera ser una infección vaginal frecuente en mujeres atendidas en un consultorio de planificación familiar, no asociándose a edad ni a factores de riesgo de ITS. La sensibilidad del método de Amsel, ocupando tres o cuatro criterios, fue $62,1 \%$ y la especificidad $92,3 \%$. Pero en la práctica clínica, como no se dispone de microscopio para la determinación de "clue cells", el diagnóstico basado en las características del flujo vaginal, la medición de su $\mathrm{pH}$ y la detección de aminas, según este trabajo, disminuye la sensibilidad del método a $21,4 \%$ manteniendo la especificidad, por lo que se recomienda que el diagnóstico se efectúe mediante el procedimiento de Nugent".

\section{Resumen}

Con el objeto de determinar la prevalencia de vaginosis bacteriana (VB) en mujeres consultantes en un Centro de Salud Familiar (CESFAM) y evaluar la utilidad diagnóstica de los criterios de Amsel, en comparación con el método de Nugent, se tomaron muestras vaginales de 100 mujeres menores de 50 años, entre abril y noviembre de 2006. Las mujeres fueron enroladas consecutivamente sin seleccionar por síntomas. Se detectó VB en $32 \%$ mujeres, sin encontrar diferencias significativas por edad $(p=0,114)$. El 87,5\% de las mujeres declaró pareja sexual única en los últimos 6 meses. El método de Amsel tuvo $62,1 \%$, de sensibilidad, $92,3 \%$ de especificidad y valores predictores positivo y negativo de 81,8 y $83,3 \%$ respectivamente. La presencia de "clue cells" fue el mejor parámetro del método de Amsel, lo que está limitado por la falta de microscopios en la práctica clínica. En conclusión, VB es una infección prevalente en la población en estudio, no se asocia a edad o actividad sexual, recomendándose para su diagnóstico el método de Nugent.

\section{Referencias}

1.- Koumans E H, Sternberg M, Bruce C, McQuillan G, Kendrick J, Sutton M, et al. The prevalence of bacterial vaginosis in the United States, 2001-2004; associations with symptoms, sexual behaviors, and reproductive health. Sex Transm Dis 2007; 34: 864-9.
2.- Leitich $\mathrm{H}$, Brunbauer M, Bodner-Adler B, Kaider A, Egarter C, Husslein P. Antibiotic treatment of bacterial vaginosis in pregnancy: a meta-analysis. Am J Obstet Gynecol 2003; 188: 752-8.

3.- Wiesenfeld H C, Hillier S L, Krohn M A, Landers D V, Sweet R L. Bacterial vaginosis is a strong predictor of Neisseria gonorrhoeae and Chlamydia trachomatis infection. Clin Infect Dis 2003; 36: 663-8.

4.- Schwebke J R. Abnormal vaginal flora as a biological risk factor for acquisition of HIV infection and sexually transmitted diseases. J Infect Dis 2005; 192: 1315-7.

5.- Ness R B, Kip K E, Hillier S L, Soper D E, Stamm C A, Sweet R L, et al. A cluster analysis 
of bacterial vaginosis-associated microflora and pelvic inflammatory disease. Am J Epidemiol 2005; 162: 585-90.

6.- Ovalle A, Martínez M A, Villagra E, Roncone E, Sandoval R, Silva R. Flora microbiana genital en embarazadas con y sin riesgo de infección. Rev Chil Obstet Ginecol 1996; 61: 5-11.

7.- Balsdon M J, Martínez M A, Céspedes P, Biotti M, Saumann A, Chávez S, et al. Vaginosis bacteriana: diagnóstico mediante tinción de Gram de muestra obtenida por dos procedimientos. Rev Chil Infect 1999; 16: 100-4.

8.- Navarrete P, Domínguez M, Castro E, Zemelman R. Evaluación de los criterios de Nugent y Amsel para el diagnóstico de vaginosis bacteriana. Rev Méd Chile 2000; 128 : 767-71.

9.- Martínez M A, Barría A, Meneses R, Oyarzún P, Sandoval J. Vulvovaginitis en la adolescencia: estudio etiológico. Rev Chil Obstet Ginecol 2003; 68: 499-502.

10.- Ovalle A, Martínez M A. Infección genital. Eghon Guzmán, Editor. Selección de temas en Ginecoobstetricia. Tomo II. $1^{\text {a }}$ ed. Santiago de Chile: Publiimpacto; 2007, p. 884-923.

11.- Centers for Disease Control and Prevention (CDC). Sexually transmitted diseases. Treatment guidelines, 2006. MMWR Morb Mortal Wkly Rep 2006; 55 (RR-11): 1-94.

12.- Amsel R, Totten P A, Spiegel C A, Chen K C, Eschenbach D, Holmes K K.
Nonspecific vaginitis. Diagnostic criteria and microbial and epidemiological associations. Am J Med 1983; 74: 14-22.

13.- Nugent R P, Krohn M A, Hillier S L. Reliability of diagnosing bacterial vaginosis is improved by standardized method of gram stain interpretation. J Clin Microbiol 1991; 29: 297-301.

14.- Forbes B A, Sahm D F, Weissfeld A (Eds). Selection of diagnostic tests. En: Bailey \& Scott's. Diagnostic Microbiology. 10th ed. St. Louis, Missouri: Mosby, Inc.; 1998, p. 60-3.

15.- Tolosa J E, Chaithongwongwatthana S, Daly S, Maw W W, Gaitan H, Lumbiganon P, et al. The international infections in pregnancy study: Variations in the prevalence of bacterial vaginosis and distribution of morphotypes in vaginal smears among pregnant women. Am J Obstet Gynecol 2006; 195: 1198-204.

16.- Eschenbach D A, Hillier S, Critchlow C, Stevens C, DeRouen T, Holmes K K. Diagnosis and clinical manifestations of bacterial vaginosis. Am J Obstet Gynecol 1988; 158: 819-28.

17.- Nelson D B, Macones G. Bacterial vaginosis in pregnancy: current findings and future directions. Epidemiol Rev 2002; 24: 102-8.

18.- Forsum U, Hallén A, Larsson P G. Bacterial vaginosis-a laboratory and clinical diagnostics enigma. APMIS 2005; 113: 153-61.

19.- Thomason J L, Gelbart S M, Anderson R J, Walt A K, Osypowski P J, Broekhuizen F F.
Statistical evaluation of diagnostic criteria for bacterial vaginosis. Am J Obstet Gynecol 1990; 162: $155-60$.

20.- Myziuk L, Romanowsky B, Johnson S C. BVBlue test for diagnosis of bacterial vaginosis. J Clin Microbiol 2003; 41: 1925-8.

21.- Swidsinski A, Mendling W, Loening-Baucke V, Ladhoff A, Swidsinski S, Hale L P, et al. Adherent biofilms in bacterial vaginosis. Obstet Gynecol 2005; 106: 1013-23.

22.- Tam M T, Yungbluth M, Myles T. Gram stain method shows better sensitivity than clinical criteria for detection of bacterial vaginosis in surveillance of pregnant, low-income women in a clinical setting. Infect Dis Obstet Gynecol 1998; 6: 204-8.

23.- Gutman R E, Peipert J F, Weitzen S, Blume J. Evaluation of clinical methods for diagnosing bacterial vaginosis. Obstet Gynecol 2005; 105: 551-6.

24.- Gardner H L, Dukes C D. Haemophilus vaginalis vaginitis; a newly defined specific infection previously classified "non specific vaginitis" Am J Obstet Gynecol 1955; 69: 962-76.

25.- Zhou X, Brown C J, Abdo Z, Davis C C, Hansmann M A, Joyce P, et al. Differences in the composition of vaginal microbial communities found in healthy Caucasian and black women. The International Society for Microbial Ecology (The ISME Journal) 2007; 1: 121-33. 